\title{
Performances Of Orthogonal Wavelet DiVISION MULTIPLEX (OWDM) SYSTEM UNDER AWGN, RAYLEIGH, AND RICEAN CHANNEL CONDITIONS
}

\author{
Mohammed Tarique \\ Department of Electrical Engineering, Ajman University of Science and Technology, \\ P.O. Box 2202, Fujairah, United Arab Emirates
}

\begin{abstract}
Orthogonal Wavelet Division Multiplexing (OWDM) has been considered as an alternative of Orthogonal Frequency Division Multiplexing (OFDM) in the recent years. OWDM has lower computational complexity and higher flexibility compared to its OFDM counterpart. The core component of OWDM is wavelet. Wavelet has been a much investigated and applied topic in digital image processing for a long time. Recently, it has drawn considerable attention of the researchers working in communication field. In this work we investigate the performances of OWDM under different channel conditions. We consider three channel conditions namely Additive White Gaussian Noise (AWGN), Rayleigh, Ricean, and frequency selective. We consider a number of wavelets namely Haar, Daubechies, Biorthogonal, Reverse Biorthogonal, Coiflets, and Symlets in OWDM design. For system model we choose Digital Video Broadcasting-Terrestrial (DVB-T). Originally DVB-T system was designed based on OFDM. In this work we use OWDM instead. The simulation results show OWDM outperforms OFDM in terms of bit error rate (BER), noise resiliency, and peak-to-average ration. The results also show that the Haar wavelet based OWDM outperforms other wavelets based OWDM system under all three considered three channel conditions.
\end{abstract}

\section{KEYWORDS}

Digital Video Broadcasting, OWDM, wavelets, AWGN, multipath, Rayleigh, Ricean, frequency selective

\section{INTRODUCTION}

Digital Video Broadcasting (DVB) was introduced by a consortium consisting of over 200 companies and regulatory bodies across Europe [1]. The main purpose was to convert analog television system into digital television system. Initially, DVB was introduced for stationary signal reception by using rooftop antenna. Extensive researches demonstrated that DVB signal could be received by mobile devices [2]. Now-a-days, DVB is enjoying an enormous growth and is a very popular technology in digital video broadcasting. Till date many commercial versions of DVB system have been introduced. Among these DVB-S, DVB-C, and DVB-T have become very popular and they are being used in satellite, cable, and terrestrial networks respectively. These technologies have been described in detail in [3-4]. Since this work is based on DVB-T we present a summary features of DVB-T here for completeness of our work.

DVB-T system operates within the existing Very High Frequency (VHF) and Ultra High Frequency (UHF) bands. Two modes of operation have been defined for the DVB-T system namely " $2 \mathrm{~K}$ mode" and " $8 \mathrm{~K}$ mode". The signal transmission of the DVB-T system is based on Moving Picture Experts Group-Two (MPEG-2). Prior to broadcasting the DVB-T signal DOI: $10.5121 /$ ijcnc. 2016.8307 
undergoes channel coding and modulation. Different levels of Quadrature Amplitude Modulation (QAM) and code rates are used in DVB-T system. The other signal processing steps done in the transmitter include multiplexing, multiplex adaptation, outer coding, outer interleaving, inner coding, inner interleaving, mapping, frame adaptation, Orthogonal Frequency Division Multiplexing (OFDM), and guard interval insertion as shown in Fig.1. Among these signal processing steps the quality of signal transmission in the DVB-T system heavily depends on OFDM.

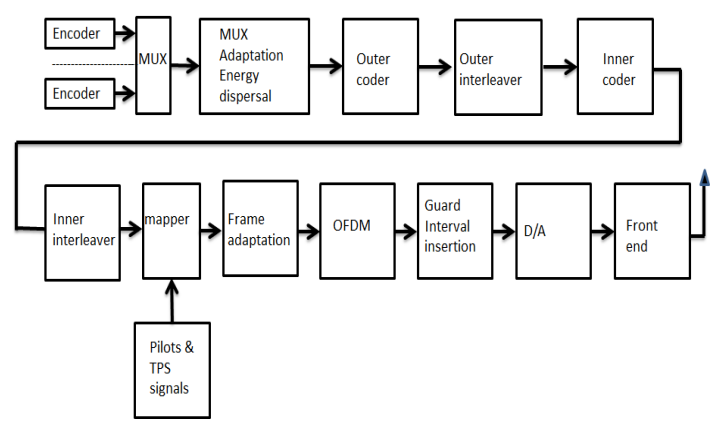

Fig. 1 Signal processing steps in the DVB-T transmitter

OFDM is a multi-carrier modulation (MCM) scheme [5-6]. The main principle of MCM is to divide the input bit stream into several parallel bit streams and then these bit streams are used to modulate several sub-carriers. OFDM employs densely spaced orthogonal sub-carriers and overlapping spectrums. Hence, it is considered as a spectrum efficient modulation scheme. Inverse Discrete Fourier Transform (IDFT) is used to implement the orthogonal signals generation in OFDM transmitter. On the other hand, Discrete Fourier Transform (DFT) is used in OFDM receiver to generate original signal from the received orthogonal signal.

OFDM has numerous advantages over other single carrier modulation schemes. The main advantage of OFDM is that it can minimize Inter Symbol Interference (ISI) originated from the multipath fading channel [7]. Hence, it can support a very high data rate. OFDM uses Fast Fourier Transform (FFT) and Inverse Fast Fourier Transform (IFFT) algorithms, which are computationally efficient. OFDM is also considered suitable for coherent demodulation. Additionally, OFDM can ensure the required Quality of Service (QoS) to the end users depending on the bandwidth. It is also considered suitable for diversity techniques (i.e., time diversity and frequency diversity).

OFDM has some disadvantages too. It requires a strong synchronization between transmitter and receiver. It is very much sensitive to phase noise and frequency offset. It is also not power efficient because the Fast Fourier Transform (FFT) algorithm and Forward Error Correction (FEC) algorithms need to be remained active all the time. It cannot utilize the advantages of the diversity gain when few sub-carriers are allotted to each user. It requires a high peak to average ration RF power amplifiers to avoid amplitude noise.

Alternatives of OFDM have been proposed in the literatures. Orthogonal Wavelet Division Multiplexing (OWDM) is one of them. The advantages of OWDM over OFDM have been addressed in [8]. Some of the advantages are worthwhile to mention here. Increase in flexibility, decrease in computational complexity, and increase in noise resiliency are top at the list. Compared to OFDM system OWDM allows more parameters to be varied. In OWDM each subcarrier can have its own coding and modulation. In OWDM more error correction can be applied to the affected sub-bands. Hence, OWDM is more resilient to frequency selective fading. 
The computational complexity in OWDM is less than that of OFDM. The computational complexity in OFDM is $\mathrm{Nlog}_{2} N$ whereas the same is only $N$ in OWDM [8].

In this work we investigate the performances of OWDM under different channel conditions. We also consider three channel models namely AWGN, Rayleigh fading, Ricean channel, and frequency selective. We consider DVB-T as a system model. Originally DVB-T system was designed based on OFDM. In this work we use OWDM instead. In order to implement OWDM based DVB-T, we replace OFDM system shown in Fig.1 with an OWDM system. In OWDM system design we consider a number of wavelets namely Haar, Daubechies, Biorthogonal, Reverse Biorthogonal, Coiflets, and Symlets. The rest of the paper is organized as follows. The basic principle of OWDM has been presented in section 2. The wavelet families and their properties have been presented in section 3. The simulation model and results have been presented in section 4 . The paper has been concluded with section 5 .

\section{RELATED WORKS}

One of the early works on wavelet-based DVB-T system has been presented in [14]. In this work the authors present a comparative study on Fourier-based OFDM and wavelet-based OFDM in DVB-T system. It is shown therein that the wavelet based OFDM out-performs the Fourier-based OFDM in terms of signal-to-noise ratio for maintaining the same bit error rate. To implement wavelet-based OFDM the authors choose Daubechies family. The authors consider two channel conditions namely AWGN and Rayleigh fading channel. It is shown that the wavelet-based OFDM out-performs Fourier-based OFDM under the both channel conditions.

In another early work [8] the author has argued that the wavelet-based OFDM is more computationally efficient compared to Fourier-based OFDM system. The author also show that wavelet-based OFDM is also more flexible and noise resilient compared to Fourier-based OFDM system. A number of wavelet families namely Symlet, Coiflet, Daubechies, and Haar have been investigated in the same work. A comparison of the performances of wavelet families has been presented in this work. It has been shown that the Haar wavelet is the best choice for DVB-T system. Only AWGN channel model has been considered in this work.

A comprehensive analysis of the performance of DWT-OFDM system has been presented in [15]. The authors also have compared these performances against the same of FFT-OFDM system under AWGN channel condition. The authors argue that DWT-OFDM does not require cyclic prefix. Hence, it is more spectrally efficient compared to FFT-OFDM. The authors also investigate other performance parameters including peak-to-average ratio (PARP). It is shown that the value of PAPR is also much less in DWT-OFDM than that in conventional FFT-OFDM. The authors conclude that the DWT-OFDM should be considered always a better candidate for DVB-T system than FFT-OFDM. The authors also argue that DWT-OFDM is more power efficient and hence it is a better choice for DVB-H. Because DVB-H has been designed for handheld devices, which have limited battery capacity.

It has been shown in [16] that the wavelets can be favourably applied in all aspects of digital wireless communication system including transceiver design, denoising, channel coding, and channel modelling. The authors conclude that DWT-OFDM has less error and lower PAPR compared to FFT-OFDM. In addition cyclic prefix (CP) can be avoided in DWT-OFDM and hence it can become more bandwidth efficient compared to FFT-OFDM. The authors argue that approximately $20 \%$ of the bandwidth of FFT-OFDM can be saved in DWT-OFDM for not using $\mathrm{CP}$. An addition bandwidth saving of $8 \%$ can also be achieved by not using pilot tones.

In [17] better wavelet packet tree (BWPT) structure has been proposed for wavelet-based OFDM system. A brute force search algorithm has been used to obtain BWPT structure. The authors 
suggests Daubechies $(\mathrm{dB})$ wavelets for implementing WOFDM. They argue that Daubechies based WOFDM reduces PAPR without any additional complexity because of BWPT structure.

A novel dual-tree complex wavelet transform (DT-CWT) scheme has been proposed for OFDM in [18]. The authors have shown that the proposed scheme shows better performance in terms of BER and PAPR than the conventional OFDM. The other advantage of DT-CWT is that it has less computational complexity and it eliminates $\mathrm{CP}$ due to the good orthogonality and time frequency localization property.

In [19] the performance of bit error rate (BER) of Discrete Fourier Transform (DFT) based OFDM has been compared with that of wavelet based OFDM system. The power spectral density (PSD) of these two different OFDM systems have also been compared in [19]. The authors have also compared the performances of the both systems by using two channel estimation techniques namely Least Square (LS) and Linear Minimum Mean Square Error (LMMSE). The simulation results show that wavelet based OFDM performs better than DFT based OFDM system. Moreover, the PSD of the wavelet based OFDM is more compact compared to its DFT based counterpart.

\section{THE OWDM SYSTEM}

Orthogonal Wavelet Division Multiplexing (OWDM) has been considered as an alternative of OFDM. Wavelet functions are used in OWDM. Now-a-days, wavelets are being commonly used in signal processing and communication systems instead of Fourier transform. The main advantage is that the wavelet can provide accurate information about the fast fluctuations of the signals in the time domain. It maps a time functions into two functions namely scale, a and translation, $b$. The continuous wavelet transform $(\mathrm{CWT})$ of a signal $\mathrm{x}(\mathrm{t})$ is defined as

$$
W(a, b)=\int_{-\infty}^{\infty} f(t) \psi_{a b}(t) d t
$$

, where $W(a, b)$ is the wavelet transform and $\psi_{a b}(t)$ is called the mother wavelet, which is defined as

$$
\psi_{a b}(t)=\frac{1}{\sqrt{a}} \psi\left(\frac{t-b}{a}\right)
$$

A scaled version of the function $\psi(t)$ with a scale factor of $a$ is defined as $\psi(t / a)$. A number of mother wavelets have been reported in the literatures. Since we focus on a digital system in this paper, we use Discrete Wavelet Transform (DWT) instead of CWT.

It has been shown in [9] that it is possible to reconstruct DWT from CWT by using a bank of high-pass filters and low-pass filters as shown in Fig.2. In this figure three sub-bands decomposition has been depicted. In real life more sub-bands decomposition is performed. The filters generate more data by convolving the input signal with filter impulse response. Hence, down sampling operation is done during decomposition by discarding the alternate samples. The synthesis process of the decomposed DWT signal is also depicted Fig.2. In order to compensate the down-sampling operation done during decomposition, up-sampling is done in wavelet synthesis by inserting zeroes in the data. The wavelet transform contains both frequency information and time information. Hence, it can convey a high data rate within each sub-band.

Contrary to the IFFT operation done in OFDM system based transmitter Inverse Discrete Wavelet Transform (IDWT) operation is done in OWDM system based transmitter. The output of the IDWT can be represented as [10] 
International Journal of Computer Networks \& Communications (IJCNC) Vol.8, No.3, May 2016

$$
s(k)=\sum_{m=0}^{\infty} \sum_{n=0}^{\infty} S_{m}{ }^{n} 2^{m / 2} \psi\left(2_{k}{ }^{m}-n\right)
$$

, where $\left\{S_{m}{ }^{n}\right\}$ are the wavelet coefficients and $\psi(t)$ is the wavelet function that is compressed $m$ times and shifted $n$ times for each subcarrier. In the receiver the inverse operation (i.e., discrete wavelet Transform) is done. The output of discrete wavelet transform (DWT) is represented as

$$
S_{m}{ }^{n}=\sum_{k=0}^{N-1} s(k) 2^{m / 2} \psi\left(2_{k}{ }^{m}-n\right)
$$

The output signal $\left\{S_{m}{ }^{n}\right\}$ is then decoded to data and applied to QAM modulator. In this work we use 4-QAM modulator.

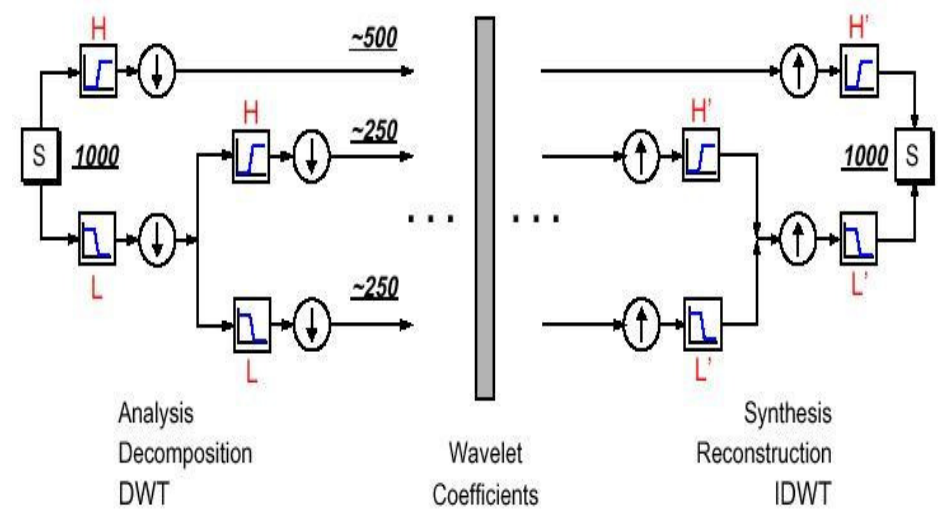

Fig. 2 The Wavelet decomposition and synthesis [9]

\section{The Wavelet Families And Their Properties}

Numerous mother wavelet and their families [11] have been proposed and used in different fields of signal processing including image processing. These mother wavelets vary in terms of symmetry, regularities, vanishing moments, and the length. In this work we focus on two important criteria namely scaling function and orthogonality (or biorthogonality). In each wavelet families there are child wavelets that are distinguished by coefficients and also by number of iterations. Since we are considering wavelet as an alternative of OFDM, we focus more on the orthogonality and biorthogonality properties. Hence, we consider only Haar, Daubechies, Biorthogonal, Reverse Biorthogonal, Coiflets, and Symlets wavelet families in OWDM simulation. These wavelet families have various degree of orthogonality compared to other wavelet families that have been reported in the literatures. The wave shapes of these investigated wavelets are shown in Fig.3.

The Haar wavelet function was proposed by Afred Haar in 1909. Among all the wavelets considered in this work the Haar wavelet is the simplest one and memory efficient. It is not continuous as shown in Fig.3. It has only one vanishing moment. The Haar function is considered only suitable for the signals that have sharp transition. The Daubechies wavelet is named after the Belgian physicist and mathematician Ingrid Daubechies. The Daubechies wavelets are orthogonal and have maximal number of vanishing moments. Among all the available Daubechies wavelets mostly used family are $\mathrm{db} 2-\mathrm{db} 20$. The index indicates the vanishing moments. For example, db2 has two vanishing moments, and $\mathrm{db} 3$ has three vanishing moments etc. The db1 has one vanishing moment and referred to as the Haar wavelet. The peculiar characteristic of the Daubechies wavelets is that they are not symmetric. 


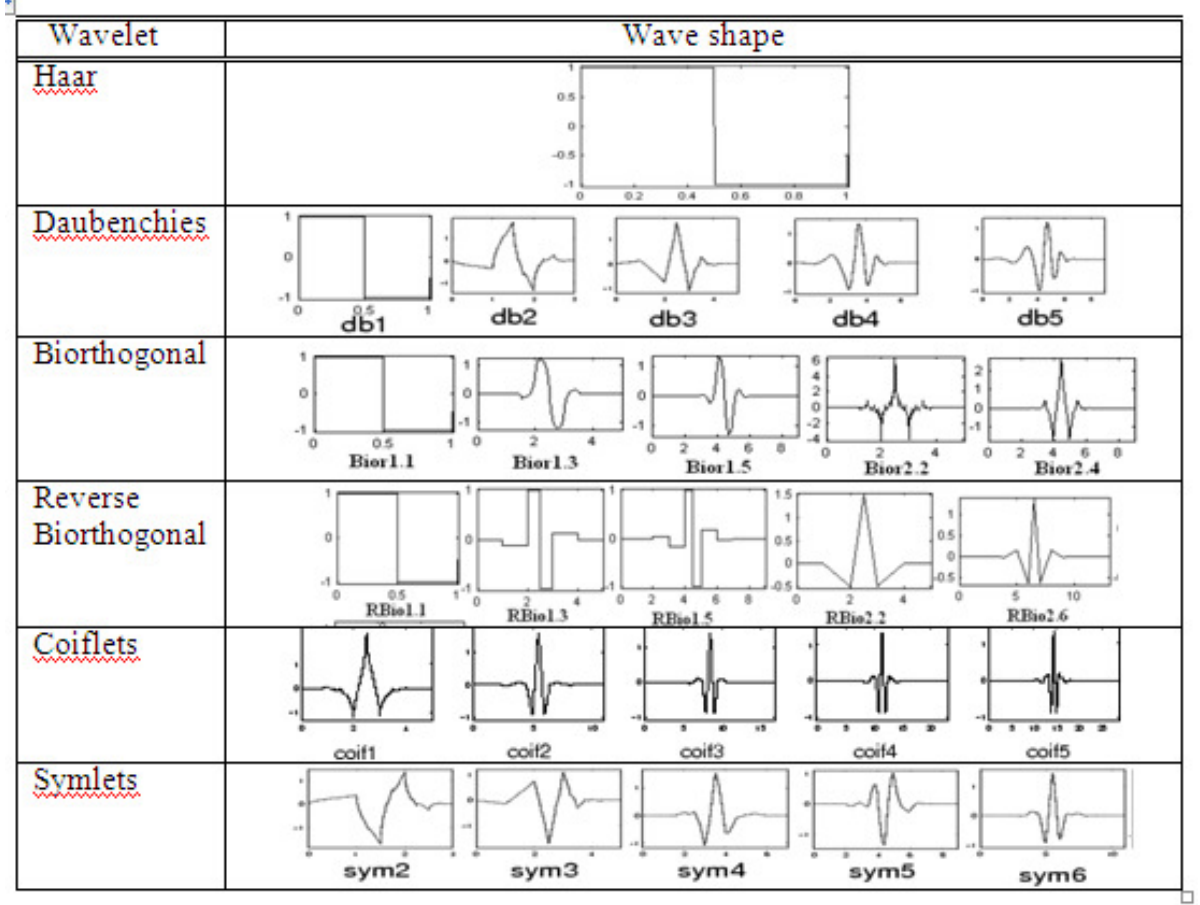

Fig.3 The wavelets and their families [12]

Biorthogonal wavelets are another popular wavelet family. They are being used in many signal processing applications due to their linear phase property. The linear phase property is achieved by maintaining symmetry in the filter coefficients. The Biorthogonal wavelets provide more degree of freedom compared to their orthogonal wavelets. The Biorthogonal wavelets are compactly supported symmetric. The Reverse Biorthogonal wavelet family is obtained from the Biorthogonal wavelet families. Both Biorthogonal and Reverse Biorthogonal wavelet families are compactly supported. The Coiflet wavelets family was introduced by Daubechies and was named in the honor of Ronald Coifman. The peculiar characteristic of the Coiflet wavelets is that they are more symmetrical than the Daubechies wavelet. The Symlet wavelets are compact, orthogonal, and continuous. These wavelets are less symmetric compared to the Daubechies wavelets. A comparison of the considered wavelets used in this work is listed in Table 1.

Table 1: The properties of wavelet families

\begin{tabular}{|c|c|c|c|c|c|c|}
\hline Property & Haar & Daubechies & Symlet & Coiflet & Biorthogonal & $\begin{array}{c}\text { Reverse } \\
\text { Biorthogonal }\end{array}$ \\
\hline Symmetry & $\mathrm{X}$ & & & & $\mathrm{X}$ & $\mathrm{X}$ \\
\hline Asymmetry & & $\mathrm{X}$ & $\mathrm{X}$ & & $\mathrm{X}$ & \\
\hline $\begin{array}{c}\text { Near } \\
\text { symmetry }\end{array}$ & & $\mathrm{X}$ & $\mathrm{X}$ & $\mathrm{X}$ & $\mathrm{X}$ & $\mathrm{X}$ \\
\hline $\begin{array}{c}\text { Arbitrary } \\
\text { number of } \\
\text { vanishing } \\
\text { moments }\end{array}$ & $\mathrm{X}$ & $\mathrm{X}$ & $\mathrm{X}$ & $\mathrm{X}$ & & \\
\hline $\begin{array}{c}\text { Compactly } \\
\text { Orthogonal }\end{array}$ & $\mathrm{X}$ & & & $\mathrm{X}$ & $\mathrm{X}$ \\
\hline $\begin{array}{c}\text { Compactly } \\
\text { Biorthogonal }\end{array}$ & & & & & & \\
\hline
\end{tabular}




\section{The Simulation And ReSults}

The DVB-T transmitter and receiver models presented in [13] have been used in this investigation. Since the number of carriers in DVB-T system is 1705, we consider 1705 4-QAM symbols as the input data to the transmitter model. We use a bandwidth of about 7.61 MHz. The carrier frequency we choose is $90 \mathrm{MHz}$. The other parameters of the simulated system are listed in Table 2.

Table 2: The Simulation Parameters

\begin{tabular}{|l|l|}
\hline \hline Parameter & Values \\
\hline Symbol Duration & $224 \mu \mathrm{Sec}$ \\
\hline Frame Size & $68 \mathrm{x} 224 \mu \mathrm{Sec}$ \\
\hline No. of Symbols & 68 \\
\hline Modulation & 4-QAM \\
\hline Wavelets & $\begin{array}{l}\text { Haar, Daubechies, Symlet, Bio-orthogona, } \\
\text { Reverse Bio-orthogoal, Coiflets }\end{array}$ \\
\hline Channel Model & $\begin{array}{l}\text { AWGN, Rayleigh, Ricean, Frequency } \\
\text { Selective (10 tap) }\end{array}$ \\
\hline Signal-to-Noise Ratio (SNR) & $0-10 \mathrm{~dB}$ \\
\hline
\end{tabular}

Initially we investigate the noise resiliency of OWDM. This is one of the main advantage of OWDM over OFDM. In order to investigate noise resiliency of OWDM, we compare the scatterplots of received symbols for OWDM and OFDM systems under AWGN channel. In both cases a signal-to-noise ration (SNR) of $10 \mathrm{~dB}$ was maintained. The scatterplots are shown in Fig. 4. This figure shows that the constellation points in OWDM based DVB-T system are very close to the ideal 4-QAM constellation points $(-1,-1),(-1,+1),(+1,-1)$, and $(-1,-1)$. On the other hand the constellation points of OFDM based DVB-T system are located far from the ideal 4-QAM constellation points.

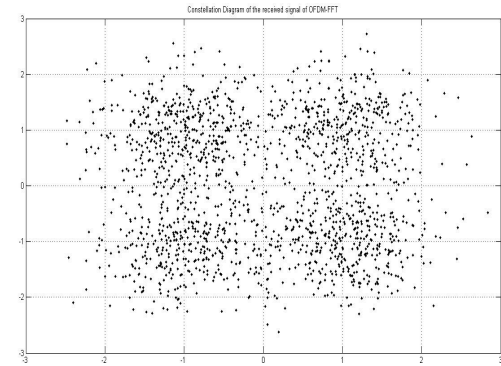

(a) OFDM constellation

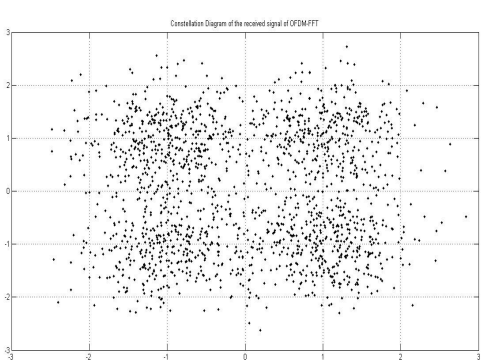

(b) OWDM constellation

Fig.4 The constellation comparison of OFDM based DVB-T and OWDM based DVB-T system. 


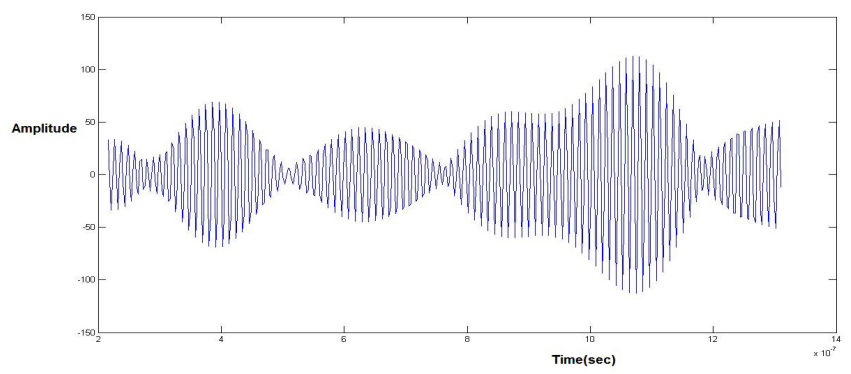

(a) OFDM based DVB-T Signal

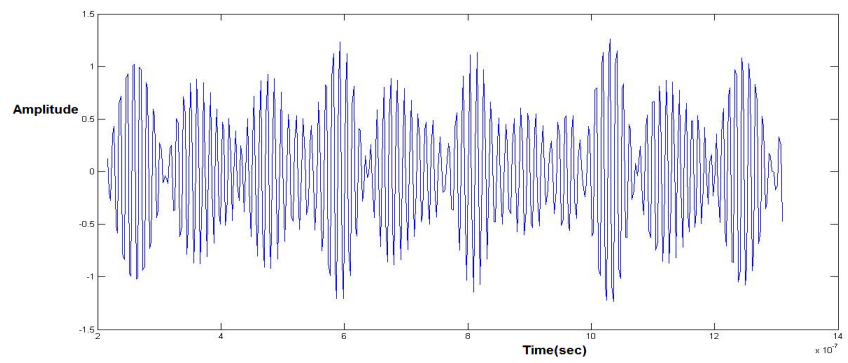

(a) OWDM based DVB-T Signal

Fig.5 Comparison of transmitted signals of OFDM based DVB-T and OWDM based DVB-T system

OWDM system solves another well-known problem of OFDM system called high PAPR. This claim is illustrated in Fig.5, which compares the transmitted signals of OFDM based DVB-T and OWDM based DVB-T. This figure shows that the PAPR for OWDM based DVB-T signal is much less than that of OFDM based DVB-T system. The symbol error rates of OFDM based DVB-T system and OWDM based DVB-T system are compared in Fig.6. The channel model considered in this case is AWGN. It is shown in the figure that wavelet based DVB-T system performs better compared to OFDM based DVB-T system irrespective of the wavelet functions. It is also shown in the figure that Haar wavelet functions outperforms other wavelet functions.

In the rest of the simulations we consider different channel models namely AWGN, Rayleigh Channel (one tap), Ricean channel, and Frequency Selective channel (10 taps). We also use different wavelets namely Haar, Daubechies, Biorthogonal, Reverse Biorthogonal, Coiflets, and Symlets. The simulation results are presented in Fig.7(a)-Fig.7(f).

The simulation results presented in Fig.7(a) show the performances of OWDM based DVB-T system based on the Haar wavelet function. It is depicted that the Haar function based DVB-T system shows the best performance under AWGN channel condition compared to other three channel conditions. But, the system performances are greatly affected by multipath conditions. However, in all cases the symbol error rate decreases with the increase in SNR. This decrease in symbol error rate is significant for AWGN, Ricean and Rayleigh fading channel except for frequency selective channel. Under the frequency selective channel condition the symbol error rate varies slightly with respect to SNR. 
International Journal of Computer Networks \& Communications (IJCNC) Vol.8, No.3, May 2016

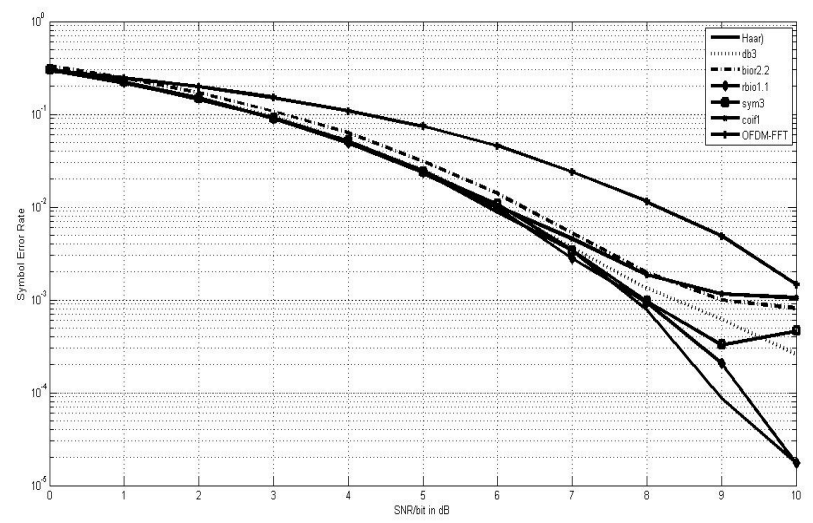

Fig.6 The performances of wavelet based DVB-T systems.

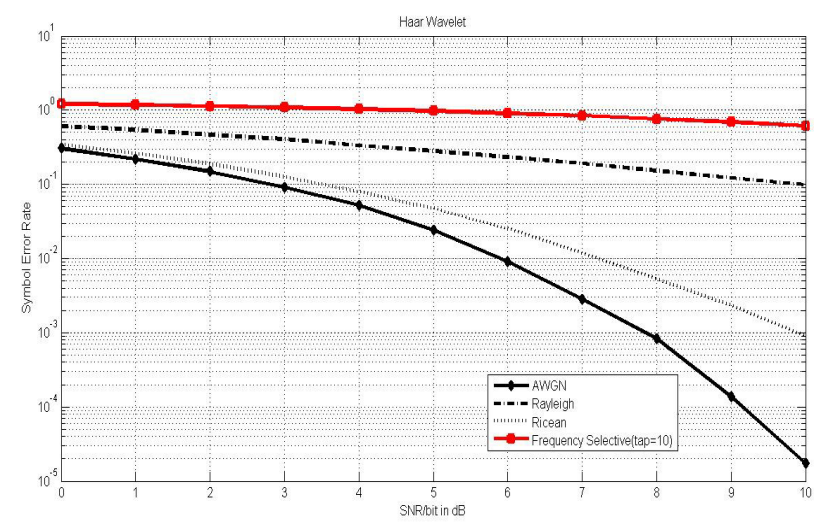

Fig.7(a) The simulation results of Haar Wavelet based DVB-T system

The simulation results of Daubechies wavelet based DVB-T system are shown in Fig.7(b). In this case the symbol error rate variation with SNR is similar to that of Haar based DVB-T system. The only difference is that symbol error rate for Haar based DVB-T system is less compared to Daubechies based DVB-T system for a given SNR.

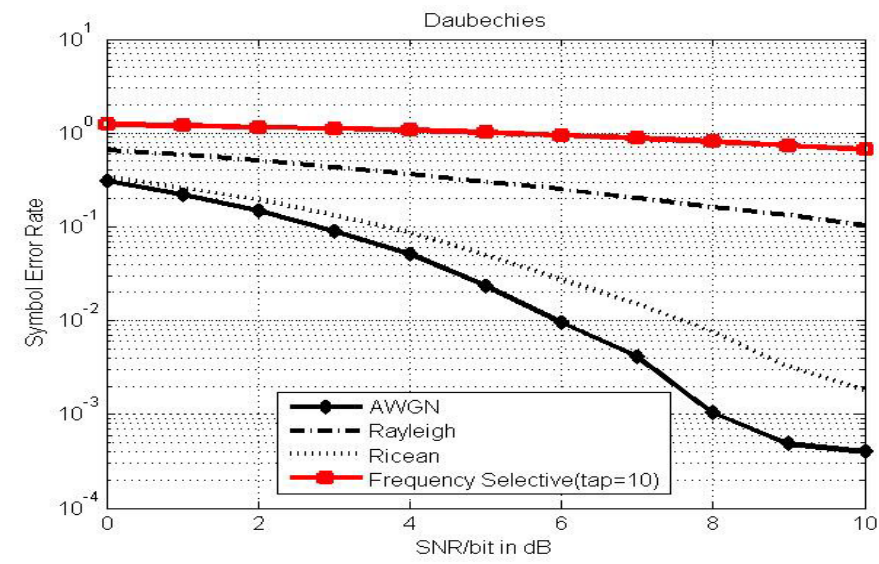

Fig.7(b) The simulation results of Daubechies Wavelet based DVB-T system 


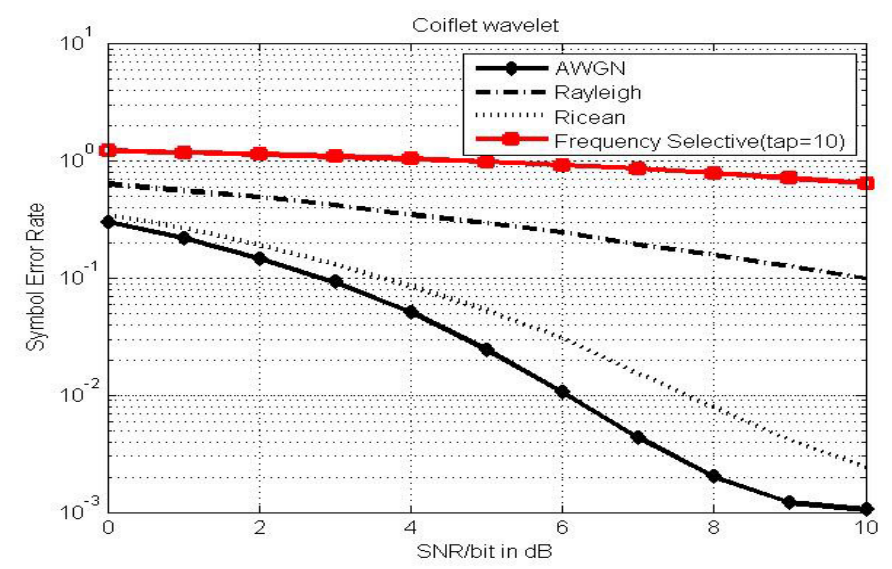

Fig.7(c) The simulation results of Coiflets Wavelet based DVB-T system

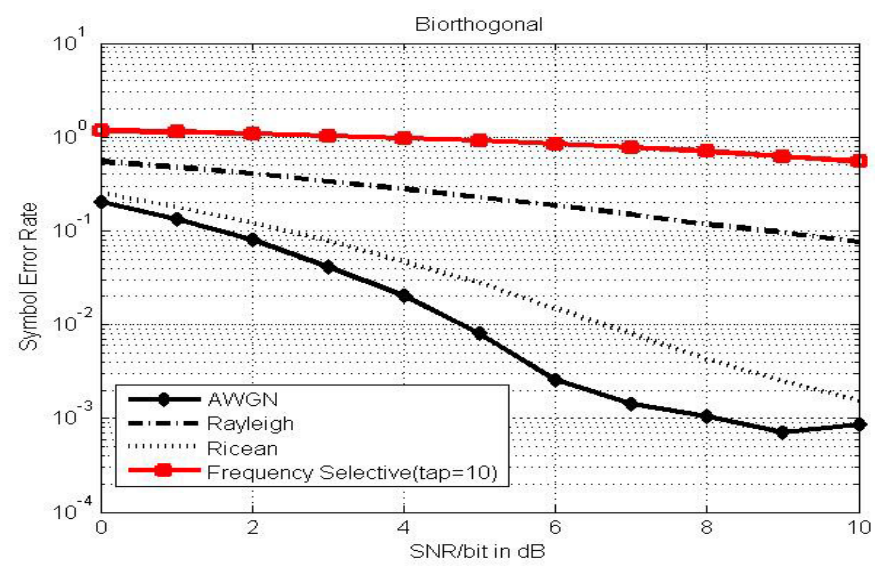

Fig. 7(d) The simulation results of Biorthogonal Wavelet based DVB-T system

The simulation results of Coiflet based DVB-T are shown in Fig.7(c). Comparing the simulation results presented in Fig.7(a) and Fig.7(b), we can conclude that Coiflet based DVB-T shows poor performance for a given SNR under all four channel conditions. Hence, Coiflet wavelet can be considered as a poor wavelet function for OWDM based DVB-T system. 


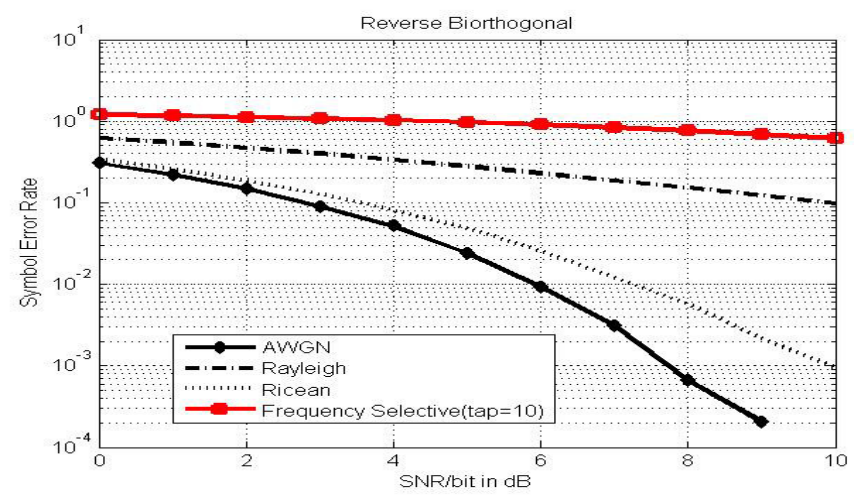

Fig.7(e) The simulation results of Reverse Biorthogonal Wavelet based DVB-T system

The simulation results for Biorthogonal and Reverse Biorthogonal wavelets functions are shown in Fig.7(d) and Fig.7(e) respectively. The symbol error rates shows that Reverse Biorthogonal based DVB-T system outperforms Biorthogonal based DVB-T system. The performances of Reverse Biorthogonal wavelet based DVB-T system are almost similar to those of Haar wavelet based DVB-T system.

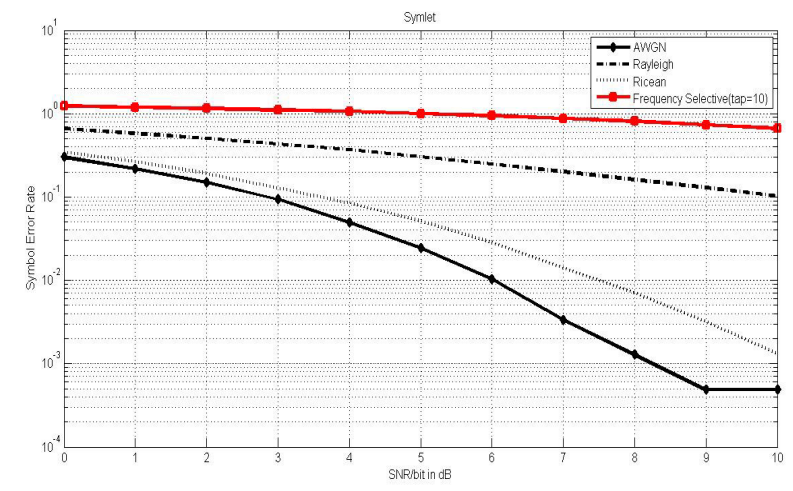

Fig. 7(f) Simulation results of Symlet Wavelet based DVB-T system

The last simulation we conducted based on Symlet wavelet function. The results are presented in Fig.7(f), which show that the performance of Symlet based DVB-T is poor compared to Haar, Daubechies, Biorthogonal, and Reverse Biorthogonal wavelets based DVB-T system. Like Coiflet wavelet we can conclude that Symlet wavelet should not be considered as a good candidate for DVB-T system.

Based on the simulation results presented in these section we can conclude that Haar wavelet and Reverse Biorthogonal wavelet are the top two candidates for OWDM based DVB-T system. Under all considered channel conditions the performances of Haar and Reverse Biorthogonal wavelets based DVB-T system are almost similar. The other three wavelets namely Symlet, Coiflet, and Biorthogonal wavelets are poor candidates for OWDM based DVB-T system. 
International Journal of Computer Networks \& Communications (IJCNC) Vol.8, No.3, May 2016

\section{Conclusions}

In this paper the performances of OWDM based DVB-T system have been investigated. Although DVB-T system was originally designed based on Fourier transform based OFDM, we consider OWDM in this work instead. We consider a number of wavelets in OWDM design. We consider four different channel conditions in order to investigate the performance. The simulation results show that OWDM based DVB-T system outperforms OFDM based DVB-T system in terms of noise resiliency and symbol error rate. In addition OWDM also reduces the peak to average ratio of OFDM signal. The simulation results show that Haar wavelet and Reverse Biorthogonal wavelet perform almost same. Hence, these two wavelet functions should be used in OWDM based DVB-T system. Still, the system performance under frequency selective fading channel is not very impressive. Hence, some advanced techniques like equalization and diversity techniques need to be incorporated in OWDM based DVB-T system in order to improve the performance under frequency selective condition. We use only six wavelets in this work. But, there are other numerous wavelet functions that have been reported in the literatures. In order to fair comparison these wavelets functions also need to be investigated. All these have been left as future works.

\section{REFERENCES}

[1] DVB Standards available at www.dvb.org

[2] Uwe Ladebusch, and Claudi A. Liss, “ Terrestrial DVB (DVB-T): A Broadcast Technology for Stationary Portable and Mobile Use", Proceedings of IEEE, Vol. 94, No. 1, January, 2006, pp. 183193

[3] Peter S., "DVB: Developing Global Television Standards for Today and Tomorrow", Technical Seminar at International Telecommunication Union (ITU), 2011

[4] Reimars, U.H., "DVB-The Family of International Standards for Digital Video Broadcasting", Proceedings of IEEE, Vol. 4, No. 1, 2006, pp. 173-182

[5] R. V. Nee and R. Prasad, OFDM Wireless Multimedia Communications, Norwood, MA: Artech House, 2000.

[6] J. A. C. Bingham, "Multi-carrier modulation for data transmission: An idea whose time has come", IEEE Communications Magazine, vol.28, no. 5, May 1990, pp.5-14.

[7] Theodore Rappaport, "Wireless Communications: Principles and Practices", Second Edition, Prentice Hall, Upper Saddle River, 2002, pp.140-141

[8] S.L. Linffot and M.K. Ibrahim, "Flexible Modulation for Digital Terrestrial Broadcasting", IEEE Electronics Letters, Vol. 42, No. 23, November 2006, pp. 1360-1362

[9] S. Mallat, "A Theory of multiresolution signal decomposition: the wavelet representation", IEEE Pattern Analysis and Machine Intelligence, Vol. 11, No. 7, 1989, pp. 674-693.1989

[10] S.L. Linffot and M.K. Ibrahim, “ Flexible Modulation for Digital Terrestrial Broadcasting”, IEEE Electronics Letters, Vol. 42, No. 23, November 2006, pp. 1360-1362

[11] G.Strang, and T. Nguyen, "Wavelets and Filter Banks", Wellesly-Cambridge Press, Massachusetts, USA, 1997

[12] G.Strang, and T.Nguyen," Wavelets and Filter Banks”, Wellesly-Cambridge Press,Massachusetts, USA, 1997

[13] OFDM Simulation using Matlab available at http://www.ece.gatech.edu/research/ labs/sarl/ tutorials / OFDM/Tutorial_web.pdf

[14] Khaizuran Abdullah and Zahir M. Hussain, "Performance of Fourier-Based and Wavelet-Based OFDM for DVB-T Systems", Proceedings of the Australian Telecommunication Networks and Applications, Christchurch, New Zealand, December 2007, pp. 475-479

[15] Vidushi Dagour , and Prabhat Patel, "Performance of Wavelet Transform based OFDM and Application to DVB system", International Journal of Innovative Research in Science,Engineering and Technology, Vol. 4, No. 8, August 2015, pp. 6760-6767

[16] M.K. Lakshmanan, H. Nikookar, "A Review of Wavelets for Digital Wireless Communication”,Wireless Personal Communication, Vol. 37, 2006, pp. 387-420

[17] Volkan Kumbasar, and Oguz Hucur, "Better wavelet packet tree structures for PAPR reduction in WOFDM System”, Digital Signal Processing, Vol. 18, No. 6, 2008, Pages 885-891 
International Journal of Computer Networks \& Communications (IJCNC) Vol.8, No.3, May 2016

[18] Mohammed H. M. Neerma, Nidal S. Kamel, Varon Jeoti,” BER Performance Analysis of OFDM System Based on Dual-Tree Complex Wavelet Transform in AWGN Channel", Proceedings of the 8th WSEAS International Conference on Signal Processing, February, 2009, Cambridge, pp. 85-89

[19] Mahesh Kuma Gupta, and S. Tiwari, "Performance evaluation of conventional and wavelet based OFDM system”, International Journal of Electronics and Communications, Vol. 67, no. 4, April 2013, pp. 348-354 